\title{
Pulmonary Tuberculosis in HIV-infected Patients Presenting with Normal Chest Radiograph and Negative Sputum Smear
}

\author{
Mounika Arrabelli ${ }^{1}$, Raghuram Reddy Adidala ${ }^{1}$, Chakrapani Chatla ${ }^{2}$, Shireesha T. ${ }^{1}$, Suryaprakash \\ Chakramahanty $^{3}$ and Malathi Jojula ${ }^{4}$ \\ 1. Department of Pharmacy, Sri Shivani Collage of Pharmacy, Warangal, Telangana State 506009, India \\ 2. World Health Organization, India Country Office, New Delhi, India \\ 3. State TB Cell, DM\&HS Campus, Koti, Hyderabad 500097, Telangana State, India \\ 4. Department of Pharmaceutical Biotechnology, Sri Shivani Collage of Pharmacy, Warangal, Telangana State 506009, India
}

\begin{abstract}
Deaths due to Tuberculosis (TB) are high among the TB-HIV co-infected patients. Among PLHIV most of the instances the sputum smear is found to be negative for MTb. Chest X-rays also don't yield much diagnostic value mainly due to the advanced immune suppressed condition. This study makes an attempt to find out the utility of alternate staining methods such as Light Emitting Diode (LED), Fluorescent Microscopy (FM) and solid culture in resource limited settings for effective diagnosis of TB among PLHIV. 2 sputum samples were collected from 102 successive presumptive TB cases, whose smear microscopy and X-Ray were negative for MTb among PLHIVs visiting ICTC at MGM hospital, Warangal, Telangana State, India. All the 102 samples were repeated with ZN microscopy and X-ray. Additionally LED and FM microscopy procedures were conducted with the NALC-NAOH concentration method. All the samples were inoculated on LJ medium for solid culture and all the positive cultures were subjected for biochemical test to identify phenotypic appearance, nitrate reduction, niacin and PNB susceptibility test for all the first line anti TB drugs as per standard guidelines. Samples found positive on microscopy were cross checked with Line probe assay (LPA). All the 102 samples collected showed negative for MTb on ZN technique and negative for pulmonary TB on chest X-rays. 15 samples were positive for MTb both on LED and FM. LPA reconfirmed the MTb in all the 15 samples tested with 11 sensitive for both INH and Rifampicin, 3 INH mono resistant and 1 Rif mono resistance patterns. Of the 102 inoculations in LJ medium, 25 culture inoculations were positive for MTb growth and also were confirmed as MTb strains based on morphological, biochemical test and growth was seen after 4th week of inoculation. Of the 25 culture positives 20 were sensitive for INH and Rif, 4 INH mono resistant and 1 resistant to all first line anti TB drugs. In smear negative and chest X-ray negative presumptive TB cases, especially in immune compromised groups such as PLHIV, it is found to be useful to subject the sputum samples to LED and FM methods and at least solid culture wherever available. These methods clearly demonstrated additional yield over conventional ZN staining which can be recommended especially in the settings where high throughput equipment such as Xpert MTB/RIFor liquid culture is not available. These proactive measures can help in early diagnosis of TB which in turn can reduce mortality due to TB among PLHIV and break the chain of transmission of TB. Recommendations: Technology such as LED or FM microscopy can be advised for effective diagnosis of TB among Presumptive TB cases in PLHIV in settings where Xpert MTB/RIF is not available.
\end{abstract}

Key words: Tuberculosis, drug sensitivity, direct AFB vs, concentrated smear microscopy, LED microscopy and FM, Line Probe Assay, culture, DST.

\section{Introduction}

Tuberculosis (TB), an infectious disease caused by Mycobacterium tuberculosis (MTB), affects the lungs

Corresponding author: Malathi Jojula, Dr., Ph.D., research field: tuberculosis and HIV-TB co-infections. and other organs of the body. TB is a leading cause of morbidity and mortality in patients with HIV/AIDS [1, 2]. Approximately one third of the world's population is infected with M. tuberculosis leading to 10 million new cases each year. India scores the highest among the TB burdened countries worldwide and in 
prevalence of multi drug resistance TB (MDR) [3]. Most of the world's tuberculosis cases occur in low-income and middle-income countries, where sputum microscopy with a conventional light microscope is the primary method for diagnosing pulmonary tuberculosis [4]. Sputum smear microscopy is the primary method for diagnosing pulmonary tuberculosis. This method is fast, inexpensive, and specific for Mycobacterium tuberculosis in high incidence areas. The main limitations of direct microscopy were noted to be; relatively low sensitivity, especially in individuals co-infected with HIV. Thus, there is a need to identify methods to improve the sensitivity of microscopy. Physical and chemical sputum processing methods including centrifugation, sedimentation, and bleach, have been studied and found to have shown promising advantages. A major shortcoming of conventional microscopy is its relatively low sensitivity compared with culture, especially in patients co-infected with HIV.

In high-income countries, fluorescence microscopy rather than conventional microscopy is the standard diagnostic method. Fluorescence microscopy is credited with increased sensitivity and lower work effort, but there is concern that specificity may be lower. We did a systematic review to summarise the accuracy of LED microscopy and fluorescence microscopy compared with conventional microscopy. Hence we like to compare different staining methods and their sensitivity and specificity. Usually the diagnosis of TB is based on the acid fast staining and culture of decontaminated sputum samples are inoculated into selective medium of Lowenstein-Jensen (LJ) medium. Though the LJ medium is gold standard it has a limitation in turnaround time which takes 2 to 8 weeks to detect MTB which delays definitive diagnosis by culture method.

Deaths occur among the TB-HIV co-infected due to Tuberculosis (TB) infection in good proportion of the cases especially in high burden countries like India. Among PLHIV (People Living with HIV/AIDS) most of the instances the sputum smear is found to be negative for MTb. Chest X-rays don't yield any diagnostic value in most of the PLHIV mainly due to the advanced immunosuppressive condition. In this study we attempted to find out the additional yield and utility of alternate staining methods such as Light Emitting Diode (LED), and Fluorescent Microscopy (FM) and solid culture against the conventional AFB staining specifically in resource poor settings.

\section{Material and Methods}

\subsection{Clinical Specimens}

This study involved 102 sputa which were collected over a period of 24 months from patients $(n=102)$ attending the Karunalaya care and treatment center, Karunapuram, and ICTC (Integrated Counselling and Testing Center for HIV) at MGM hospital, Warangal City in Telangana State, India.

\section{Inclusion criteria}

- The patients chosen for this study were positive for any of the following clinical symptoms: fever, cough with or without expectoration of sputum, weight loss, night sweats, haemoptysis, reduced appetite, had $\mathrm{X}$-ray for TB and any past history of TB.

- HIV reactive suspected with TB symptoms was included in the study.

Exclusion criteria

- Extra-pulmonary TB

- PLHIV without symptoms of TB

\subsection{Sample Type}

Blood ( $2 \mathrm{ml}$ ) and 2 sputum (early morning and spot) samples were collected with patient's informed consent.

\subsection{Sample Collection}

Approximately 500 outpatients attended the ICTC centre, ART centre and HIV care and treatment centre which are attached and supporting MGM hospital Warangal for diagnosis, treatment for HIV patients. Collection of samples was from 2013 December-2015 
December the study was supported by DST-SERB from New Delhi.

\subsection{Methods}

All the clients visiting ICTC at MGM Hospital, Warangal were tested for HIV as per NACO guidelines. Those who were found to be reactive for HIV at ICTC were referred to ART Center and were provided with CD4 and CD8 counts as per NACO guidelines. The PLHIV were provided with further care and support services at Karunalayam care and treatment centre, Karunapuram. The PLHIV visiting Karunalayam were screened for symptoms of TB proactively and those who were having any one of the designated symptoms of TB as per RNTCP guidelines were provided with chest $\mathrm{x}$-ray and sputum smear microscopy.

All the consecutive out patients among PLHIV with symptoms of TB attending the ICTC were enrolled in the study. They were counselled, explained about enrolment in the study and the samples of sputum and blood were collected. Specimens were transported as soon as possible after collection to the laboratory at Sri Shivani College of Pharmacy which has a Biosaftey Level II Laboratory. If delay is unavoidable, the specimens were refrigerated to inhibit the growth of unwanted micro-organisms. When pulmonary TB was suspected, specimens originating from the respiratory tract were collected, i.e., sputum and subjected for the diagnosis of pulmonary TB, as per the recommendations endorsed by the International Union against Tuberculosis and Lung Disease (IUATLD) and the World Health Organization (WHO) [5]. Early morning sputum specimens (not saliva) obtained after a deep, productive cough on non-consecutive days, apart from the number of samples collected, sputum specimens were classified in the laboratory with regard to their quality, i.e., bloody, purulent, muco-purulent or salivary. Specimens were scored as saliva or sputum on the basis of visual examination. Suspects were requested to give another specimen in case of saliva specimens. Spot sputum samples were collected where the chances of missing early morning samples was expected. For this study we had enrolled 102 presumptive TB cases among PLHIV and subjected to direct sputum microscopy (AFB) and compared with concentrated smear microscopy LED and FM to compare sensitivity and specificity of the techniques.

\subsection{Smear Preparation and Microscopic Observation}

- Staining procedures: One spot morning sputum sample was collected in a sterile container. Smear was prepared from yellow purulent portion of the sputum using a sterile bamboo stick. The smear was spread evenly, air-dried for $15 \mathrm{~min}$, and heat fixed by placing the slide over a plate for $3 \mathrm{~min}$. Three direct smears were prepared for staining by auramine $\mathrm{O}$ staining technique and examined under fluorescent microscope, other part of slide is stained and examined under LED fluorescence microscope and third slide is used for staining by $\mathrm{ZN}$ staining.

- Direct Method by AFB: Fix the smear of the specimen over the glass slide, either by heating or alcohol fixation and pour carbol fuschin over smear and heat gently until fumes appear. Do not overheat and allow it to stand for $5 \mathrm{~min}$, and then wash it off with water. Pour $20 \%$ sulphuric acid, wait for one minute and keep on repeating this step until the slide appears light pink in color. Wash off with water. Pour methylene blue, wait for two min, again wash with water. Allow it to air dry and examine under oil immersion lens.

- Concentration Method: The specimens were processed by a standard NALC-NaOH digestion-decontamination method; briefly, an equal volume of $2 \% \mathrm{NaOH}$ and $1.45 \%$ sodium citrate containing $0.5 \%$ NALC were added to each tube. The contents within the tubes were then mixed by vortexing and then incubated at room temperature for $15 \mathrm{~min}$. The tubes were then shaken by hand at regular intervals. Phosphate-buffered saline ( $\mathrm{pH}$ 6.8) were added up to $45 \mathrm{ml}$ and then centrifuged at $3,000 \mathrm{X}$ g for $15 \mathrm{~min}$. The supernatant were carefully poured off, the resulting 
sediments were then re-suspended in $1.5 \mathrm{~mL}$ of phosphate-buffered saline, and the suspensions were used to prepare smear (concentrated smear). The NALC-NAOH concentration method was used in LED and FM. Chest Radiograph was provided to all the 102 presumptive TB cases. All the samples were inoculated on LJ Medium for solid culture and all the positive cultures were subjected for biochemical test to identify phenotypic appearance, nitrate reduction, niacin and PNB susceptibility test for all the first line anti TB drugs as per standard guidelines.

\subsection{Culture Method for Isolation and Identification of} M. tuberculosis

All samples included in this study were decontaminated by N-Acetyl L-cystiene-Sodium hydroxide method (NALC-NaOH). Briefly, the sample (2-5 $\mathrm{mL}$ ) was decontaminated by mixing properly with the equal volume of $2 \% \mathrm{NaOH}$ NALC powder and Tri sodium citrate solution was allowed to stand for $15 \mathrm{~min}$ at $37{ }^{\circ} \mathrm{C}$. To this solution sterile distilled water is added upto the neck of the McCartney bottle. The sample was then centrifuged at 3,000 rpm for $15 \mathrm{~min}$, and the pellet was subjected for inoculation into L-J medium to isolate M. Tuberculosis.

Culture Media: The definite diagnosis of tuberculosis demands that M. tuberculosis is to be recovered on culture media and identified using differential in vitro tests. Many different media have been devised for cultivating tubercle bacilli and three main groups can be identified, viz egg-based media, agar-based media and liquid media.

\begin{tabular}{|lc|}
\multicolumn{2}{c}{ Ingredients } \\
Mineral salt solution with malachite green \\
Potassium dihydrogen phosphate anhydrous (KH2PO4) \\
Magnesium sulphate anhydrous & $2.4 \mathrm{~g}$ \\
Magnesium citrate & $0.24 \mathrm{~g}$ \\
Asparagine & $0.6 \mathrm{~g}$ \\
Glycerol (reagent grade) & $3.6 \mathrm{~g}$ \\
Malachite green, 2\% solution* & $12 \mathrm{~mL}$ \\
$*$ Malachite green solution & $20 \mathrm{~mL}$ \\
\end{tabular}

\subsection{Preparation of Lowenstein-Jensen Medium}

Dissolve the dye in distilled water completely. Filter and store in refrigerator. Dissolve the ingredients in order in about $300 \mathrm{~mL}$ distilled water by heating. Add glycerol, malachite green solution and make up $600 \mathrm{~mL}$ with distilled water. This solution should be sterilized by autoclaving at $121^{\circ} \mathrm{C}(15 \mathrm{psi})$ for $30 \mathrm{~min}$. Cool to room temperature. If required, this solution may be stored in the refrigerator.

\subsection{Preparation of Complete Medium}

The following ingredients are aseptically pooled in a large, sterile flask and mixed well:

- Mineral salt solution with malachite green 600 $\mathrm{mL}$

- Homogenised eggs (25-30 eggs, depending on size) $1,000 \mathrm{~mL}$

- The complete egg medium is distributed in 6-8 $\mathrm{ml}$ volumes in sterile universal containers and the caps tightly closed and inspissated without delay to prevent sedimentation of heavier ingredients.

The LJ medium should be dated and stored with the batch number in the refrigerator and can keep for up to 4 weeks if the caps are tightly closed to prevent drying of the medium.

\subsection{Drug Susceptibility Tests}

There are three general methods used for determining drug susceptibility of mycobacterium: the proportion method, absolute concentration method (MIC method) and the resistance ratio method. When properly standardized and performed, all three methods have been shown to be equally satisfactory. In India, proportion method is advised since large numbers of laboratories have standardized this method for DST.

\section{Results}

A total of 1,893 PLHIV were interviewed consisting of 859 males and 980 females before obtaining 102 PLHIV with TB symptoms and negative for TB in 
smear microscopy and X-Ray in the facility where recruitment into the study was done. The sample did not differ much in age at recruitment into the study, gender or by occupation though the population was little skewed towards married subset and middle income subgroup (Table 1).

Of the 102 patients whose sputum samples were tested for tuberculosis infection by various methods $98 \%$ were negative by $\mathrm{ZN}$ smear and X-ray while the positivity increased significantly by FM and LED microscopy methods (Table 2).

Of the 17 Sputum smear positive cases, 15 Samples were smear negative on $\mathrm{ZN}$ Technique and 2 were smear positive on $\mathrm{ZN}$ microscopy. The 15 samples were cross checked with Line probe assay (LPA) of which 11 were INH and Rif sensitive MTb, 3 were INH mono resistant $\mathrm{MTb}$ and 1 was Rif mono resistant $\mathrm{MTb}$ cases.

Table 1 Demographic features of the study population.

\begin{tabular}{ll}
\hline Demographic Feature & $(\mathrm{n}=102)$ \\
\hline Age in years & $12(12 \%)$ \\
$10-20$ & $30(29 \%)$ \\
$20-30$ & $30(29 \%)$ \\
$30-40$ & $23(23 \%)$ \\
$40-50$ & $07(7 \%)$ \\
$>50$ & \\
\hline Gender & $48(47 \%)$ \\
Male & $54(53 \%)$ \\
Female & $34(33 \%)$ \\
Socio Economic Status & $68(67 \%)$ \\
Lower & $0(0 \%)$ \\
Middle & $81(79 \%)$ \\
Upper & $14(14 \%)$ \\
Marital Status & $07(7 \%)$ \\
Married & $19(18 \%)$ \\
Unmarried & $11(11 \%)$ \\
Widowed & $61(60 \%)$ \\
Occupation & $11(11 \%)$ \\
Housewife & Farmer
\end{tabular}

Table 2 Positivity of TB by different methods of TB diagnosis used.

\begin{tabular}{llll}
\hline$(\mathrm{n}=102)$ & Positive for TB & Negative for TB & \\
\hline ZN & $2(2 \%)$ & $100(98 \%)$ & - \\
FM & $17(17 \%)$ & $85(83 \%)$ & $P<0.05$ \\
LED & $17(17 \%)$ & $85(83 \%)$ & $P<0.05$ \\
X-Ray & $2(2 \%)$ & $100(98 \%)$ & $P=1.00$ \\
\hline
\end{tabular}

Of all 102 samples put on solid culture, 26 were had growth of MTb and 76 had no growth. When DST (Drug Sensitivity Testing) was performed on 26 culture positive samples, 21 of them were found to be sensitive for all first line anti TB drugs, 4 had INH mono resistance and 1 case had resistance to all first line anti TB drugs. This is consistent with the LPA results with additional yield through culture and DST.

Thus, it is clearly demonstrated that there is higher yield of MTb positivity through solid cultures and LPA over LED microscopy and FM microscopy which in turn found to be advantageous compared to $\mathrm{ZN}$ microscopy in resource limited settings where $\mathrm{X}$ pert MTB/Rif or Liquid culture could not be performed.

\section{Discussion}

In this study, we observed that FM and LED microscopy using concentrated methods had increased the sensitivity of microscopy compared with the direct smear. Sputum smear microscopy methods are to be considered as a simple, rapid and inexpensive technique which is highly specific in areas with a very high prevalence of tuberculosis. However, sputum smear microscopy has significant limitations in its performance. The sensitivity is grossly compromised when the bacterial load is less than 10,000 organisms/mL sputum sample. It also has a poor track record in extra-pulmonary tuberculosis, paediatric tuberculosis and in patients co-infected with HIV and tuberculosis [6, 7]. Due to the requirement of serial sputum examinations, some patients who do not come back for repeated sputum examinations become "diagnostic defaulters" [5]. Some do not come back for results, and are lost to treatment and follow up. During the study it was observed that limited resources, large numbers of samples, all combined together often reduce the observation time per slide to less than $60 \mathrm{sec}$, and this also contributes to reduction in the sensitivity of the test. Therefore, techniques for optimization of smear microscopy are under active investigation. There has been an attempt to reduce diagnostic errors by assessing the feasibility of diagnosing pulmonary 
tuberculosis by collecting two sputum samples on a single day (1-day protocol), and comparing this protocol with the national policy of collecting samples on consecutive days (2-day protocol). It was felt that since the 2-day protocol did not show a statistically significant difference in diagnostic performance compared with the 1-day protocol, the latter may be adopted as an alternative protocol, particularly for patients who are more likely to default [8].

We did a systematic review to assess the ability of different processing methods to improve the sensitivity of microscopy. By searching many sources, we identified 83 studies. Overall, by comparison with direct smears, the results suggested that centrifugation with any of several chemical methods (including bleach) is more sensitive, that overnight sedimentation preceded by chemical processing is more sensitive, and that specificity is similar. There were insufficient data to determine the value of sputum processing methods in patients with HIV infection. Operational studies are needed to determine whether the increased sensitivity provided by processing methods is sufficient to offset their increased cost, complexity, and potential biohazards, and to examine their feasibility.

Despite its drawbacks, direct microscopy remains the cornerstone of TB diagnosis in developing countries, particularly in India. Direct microscopy has low sensitivity, as described in a review of 14 studies [6]. However, in this study, direct microscopy showed a relative increase in sensitivity. This finding cannot be extrapolated; however, as it was likely the result of the setting in which this study was conducted: a research laboratory with greater time resources than routine diagnostic laboratories, particularly government-owned or public health facilities.

\section{Conclusions}

In smear negative and chest $\mathrm{X}$-ray negative presumptive TB cases, it is found to be useful to subject the samples from PLHIV to LED or FM microscopy methods and solid culture or LPA if liquid culture is not available. These methods can be used for early and rapid detection and identification of tuberculosis infections from direct sputum with better sensitivity so that chances of missing the cases is reduced.

Conflict of interest: None declared.

\section{Acknowledgments}

We thank P. Lavanya, P. Mamatha, CH. Prashanthi and A. Babu for the technical assistance during implementation of the Project and Mr. Harish for the support rendered in statistical analysis. Dr. Malathi Jojula is thankful to DST SERB for a Young scientist Fellowship. Financial support to M.J. from DST SERB is acknowledged.

\section{References}

[1] World Health Organization. Global tuberculosis report 2014. WHO/HTM/TB2014.08. Geneva, World Health Organization, 2014.

[2] Migliori, G. B., Dara, M., and de Colombani, P., et al. 2012. "Multidrug-resistant Tuberculosis in Eastern Europe: Still on the Increase?" Eur. Respir. J. 39: 1290-1.

[3] RNTCP Annual Report, Government of India, 2015.

[4] The Global Plan to Stop TB, 2011-2015, World Health Organization.

[5] Luelmo, F. 2004. What is the role of sputum microscopy in patients attending health facilities? Frieden $\mathrm{T}$, editor. Toman's tuberculosis: case detection, treatment, and monitoring-questions and answers, 2nd ed. Geneva: World Health Organization, 7-13.

[6] Falzon, D., Gandhi, N., and Migliori, G. B., et al. 2013. "Resistance to Fluoroquinolones and Second-line Injectable Drugs: Impact on Multidrug-resistant TB Outcomes." Eur. Respir. J. 42: 156-68.

[7] Migliori, G. B., Sotgiu, G., and Gandhi, N. R., et al. 2013. "Drug Resistance beyond Extensively Drug-resistant Tuberculosis: Individual Patient Data Meta-analysis.” Eur. Respir. J. 42: 169-79.

[8] Perkins, M. D. 2000. "New Diagnostic Tools for Tuberculosis." Int. J. Tuberc. Lung Dis. 4 (12): S182-8. 